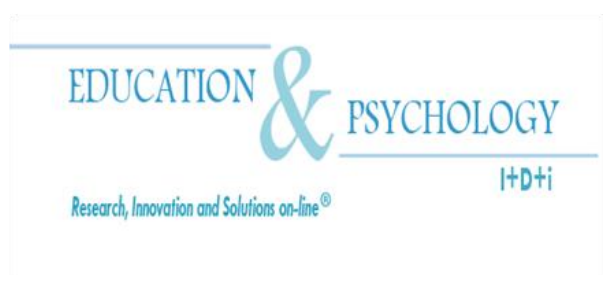

\title{
Desarrollo de la Memoria de Trabajo y Desempeño en Cálculo Aritmético: un Estudio Longitudinal en Niños
}

\author{
Magdalena López
}

Centro Interdisciplinario de Investigaciones en Psicología Matemática y Experimental (CIIPME-CONICET). Universidad Católica Argentina. Entre Ríos

\section{Argentina}

Correspondencia: Magdalena Lopez. Facultad Teresa de Avila. Buenos Aires 239. (3100)Entre Ríos. Argentina. E-mail: magdalenaglopez@gmail.com 


\section{Resumen}

Introducción. Este estudio ha tenido por objetivo investigar la relación entre el desarrollo de la memoria de trabajo y el desempeño en actividades aritméticas.

Método. Se realizó un estudio longitudinal con una muestra de 90 niños, a los que se evaluó durante tres años consecutivos (i.e., primero, segundo y tercer año de escuela primaria) respecto al desempeño en cada componente de la MT (i.e., (i) bucle fonológico, (ii) agenda visoespacial y (iii) ejecutivo central y a su conocimiento en cálculo aritmético.

Resultados. Los resultados indican relación entre el desarrollo progresivo de la capacidad de memoria de trabajo y los desempeños en aritmética a través del tiempo. Evidencia de ello es que el grupo con mayor desempeño en memoria de trabajo obtiene mejores puntajes en cálculo, durante los tres años de estudio.

Discusión y Conclusiones. La discrepancia en los puntajes en cálculo se incrementa según pasan los años debido a la incidencia que tiene la memoria de trabajo en las estrategias utilizadas para la resolución de problemas aritméticos.

Palabras Clave: memoria de trabajo, cálculo, conocimientos aritméticos, niños. 


\title{
Development of working memory and performance in arithmetic: a longitudinal study with children
}

\begin{abstract}
Introduction. This study has aimed to investigate the relationship between the development of working memory and performance on arithmetic activities.

Method. We conducted a 3-year longitudinal study of a sample of 90 children, that was followed during the first, second and third year of primary school. All children were tested on measures of WM components (i. e., (i) phonological loop, (ii) visuospatial sketchpad and (iii) central executive) and arithmetic.
\end{abstract}

Results. Results indicate the relationship between the progressive development of working memory capacity and performance in arithmetic through time. Evidence of this is that the group with higher working memory performance gets better scores in calculus, during the three years of study.

Discussion and Conclusion. The discrepancy in scores in calculation increases as the years because of the impact it has on working memory strategies used for solving arithmetic problems.

Keywords: working memory, calculation, arithmetic knowledge, children 


\section{Introducción}

Los avances en investigaciones neuropsicológicas nos permiten conocer que la maduración estructural de las diversas regiones encefálicas y de sus conexiones constituye una condición necesaria para la adquisición de habilidades cognitivas. El desarrollo de las estrategias de memoria es un proceso que comienza en el niño desde sus primeros años, flexibilizándose y ampliándose progresivamente, a medida que adquiere más conocimientos y experiencias.

Las actividades que el niño debe afrontar dentro de la escolarización, sobre todo en los primeros años, donde se ponen en juego constantemente la resolución de tareas y los problemas cognitivos, son un factor clave para el desarrollo de la conducta estratégica, permitiendo una progresiva adquisición y extensión de la misma. Comprender por qué las personas aprenden determinadas nociones a determinadas edades y cuáles son los procesos involucrados posibilita el desarrollo de métodos más eficaces, adecuados y favorables de enseñanza.

\section{La memoria de trabajo}

El modelo multicomponente de Baddeley y Hitch (1974) constituye la aproximación conceptual más ampliamente aceptada para explicar el fenómeno de la memoria de trabajo. Se concibe como un sistema cerebral que proporciona almacenamiento temporal y permite la manipulación de la información necesaria para tareas cognitivas complejas, interviniendo en forma relevante en los aprendizajes elementales (Baddeley, 1992). En dicho modelo encontramos un controlador atencional, al que llamaron el ejecutivo central, que funciona como enlace entre la memoria a largo plazo y dos sistemas subsidiarios, el bucle fonológico y la agenda visoespacial. Estos sistemas combinan la capacidad de almacenamiento temporal de información con un grupo activo de procesos de control, que permite que la información sea registrada intencionalmente y mantenida dentro del subsistema.

La memoria de trabajo sigue un curso de desarrollo postnatal que comienza en la temprana infancia y continúa durante la adolescencia (Luciana, Conklin, Hooper, y Yarger, 2005; Zald y Iacono, 1998). Específicamente, se ha demostrado que la estructura básica de la memoria de trabajo (según el modelo de Baddeley y Hitch, 1974) está presente desde los 6 años de edad y cada componente del modelo aumenta su capacidad hasta la adolescencia (Gathercole, Pickering, Ambridge, y Wearing, 2004). 


\section{Funciones de la memoria de trabajo}

La memoria de trabajo, como un sistema, no es necesaria en todas las operaciones cognitivas. Existen operaciones que realizamos diariamente de una forma bastante automática, con poca o ninguna necesidad de la memoria de trabajo, que quedan guardados como patrones motores. Por este motivo, las rutinas básicas pueden ser recuperadas casi sin esfuerzo de la memoria y permiten realizar varias actividades a la vez. Sin embargo, la memoria de trabajo sí es necesaria cuando la actividad meta entra en conflicto con tendencias automáticas, teniendo que abocarse al control necesario para frenarlas.

Para Unsworth y Engle (2007), la memoria de trabajo es necesaria: 1) en el control o la anulación de tendencias de respuesta automática y 2) en el mantenimiento y la recuperación de información de la memoria a largo plazo. El mantenimiento es necesario para sostener a la nueva información en un estado de actividad, particularmente ante la presencia de distracciones internas o externas. Este sistema está limitado por la cantidad de información que puede ser mantenida en un momento dado. Para poder mantener la información se necesita que la atención no se deje capturar por distractores que hagan perder el acceso a la tarea que debe realizarse. Unsworth y Engle sostienen que las diferencias individuales en la memoria de trabajo derivan de diferencias en las habilidades, tanto de mantener activa la información como de recuperar la información pertinente en función de la presencia de información irrelevante o distractores. En situaciones en que la información no puede ser mantenida de forma activa, la recuperación de la información pertinente, de la memoria a largo plazo, se logra a través de la discriminación. En este proceso de recuperación, las claves contextuales activan la información relevante para la tarea a realizar. Las personas con bajos desempeños en la memoria de trabajo tienen menos recursos para realizar la discriminación entre información relevante e irrelevante, incluyendo muchas representaciones en sus conjuntos de búsqueda (Unsworth y Engle, 2007).

\section{Los Conocimientos Aritméticos y las Estrategias de Resolución}

Las operaciones aritméticas consisten en procesos que permiten manipular simbólicamente datos, que resultarían difíciles de manipular de forma real. Dichas operaciones requieren que se haya adquirido el concepto de número, la función simbólica, la comprensión de la reversibilidad, así como una correcta percepción del tiempo y de la orientación espacial. 
La aritmética requiere de varios procesos cognitivos y de estrategias de resolución dependiendo, entre otros, del tipo de operación y de los símbolos numéricos (Lee y Kang, 2002). Las estrategias empleadas por los niños para la resolución de operaciones aritméticas varían en función de la edad, en busca de la rentabilidad. Al principio, las preferidas son las que se acercan más a lo manipulativo, pero pronto, en la medida en que el niño se va sintiendo más cómodo cuando opera en el ámbito simbólico con los números, se tiende a escoger y, por lo tanto, se utilizan con más frecuencia las estrategias que resultan más eficaces en términos de tiempo.

Geary y Brown (1991) plantean que en lo que respecta a las operaciones aritméticas simples, los niños las resuelven con algunas estrategias básicas. Una de las primeras estrategias consiste en representar los dos sumandos mediante objetos o dedos y enumerar ordenadamente ambos conjuntos (contar todo). La segunda estrategia aparece cuando el niño ve que contar a partir de uno de los sumandos (en virtud del principio de cardinalidad se sabe que el cardinal final equivale a todos los elementos del conjunto) ahorra tiempo (contar desde). La tercera estrategia aparece cuando el niño descubre que el mayor ahorro se produce cuando se cuenta desde el sumando mayor. Una cuarta estrategia consiste en la descomposición de los problemas en problemas más simples (Siegler, 1987), y una quinta estrategia es la de recuperación, en la que la respuesta se obtiene directamente de la memoria a largo plazo (Siegler y Shrager, 1984). Debemos decir que la operación de resta está estrechamente relacionada con la de suma ya que es un proceso basado en la reversibilidad, es prácticamente imposible calcular una resta si no pueden realizarse operaciones de suma.

Gracias a la comprensión y aplicación de los conceptos básicos a las operaciones, el niño va acumulando más y más información relativa a los números y sus propiedades y cómo operar con ellos, lo que a su vez facilita la construcción del pensamiento matemático. Existiría un patrón madurativo de inicio frontal que progresivamente se especializaría a un procesamiento parietal, una vez automatizada la relación entre los símbolos numéricos y las magnitudes que representan (Ansari, Gracia, Lucas, Halmon y Dhital, 2005). Los estudios con neuroimágenes dan pruebas de que la activación de la corteza prefrontal parecen estar vinculadas a funciones relacionadas con la memoria de trabajo, como el mantenimiento provisional de los resultados intermedios, la planificación y la ordenación temporal de los componentes de las tareas, o la comprobación de resultados y la corrección de errores (Müller y Knight, 2006). 
La participación de la región prefrontal en los circuitos neurales del cálculo se relacionaría con el almacenamiento y la recuperación de la información necesaria para la resolución de las tareas de cálculo. Parece ser que el empleo de estas estrategias es lo que permitiría a las personas dotadas para el cálculo, la resolución de tareas aritméticas de forma rápida y eficiente (Serra-Grabulosa, et.al., 2010). Haciendo una revisión de los trabajos con niños que experimentan dificultades en el aprendizaje de las matemáticas, se puede ver que no muestran deficiencias graves al inicio de su escolaridad, en los conceptos y habilidades matemáticas informales que sustentan el concepto de número. En muchos casos su desempeño es cualitativamente similar al de cualquier niño sin dificultades de aprendizaje. En cambio sus deficiencias son importantes en dos áreas nucleares de la matemática formal: la recuperación rápida de hechos numéricos y las habilidades para resolver problemas complejos que implican operaciones básicas.

Según Siegler (1984) la información sobre los hechos aritméticos básicos se almacena en la memoria en forma de "nodos", que representan tanto a los problemas como a las respuestas. Esta relación entre problemas y respuestas es a veces correcta y en otras ocasiones incorrecta. Este autor postula que cuando el niño se enfrenta a un problema de cálculo, en primer lugar, intenta recuperar la respuesta desde la memoria. Si la respuesta no supera el criterio de confianza interno del niño, éste pone en práctica estrategias de comprobación que tienen mayor probabilidad de ser correctas que las de recuperación. En general los niños más pequeños o sea con menos experiencia, utilizan estrategias de comprobación más frecuentemente que los más mayores, porque relacionan más respuestas con un problema y ninguna de las respuestas reúne los criterios de confianza. Pero puesto que las relaciones entre los problemas y la respuesta correcta son gradualmente más fuertes, las estrategias de comprobación son reemplazadas por las de recuperación directa. Esto se debe a que al comienzo del aprendizaje aritmético el niño posee una escasa fuerza asociativa internodal, por lo que frecuentemente debe recurrir a asociaciones visuales, conjeturas u otras estrategias.

A medida que se producen respuestas acertadas se aumenta la fuerza asociativa entre nodos de hechos aritméticos y nodos de respuestas, y se va produciendo la automatización de las operaciones aritméticas elementales, lo cual supone un importante ahorro cognitivo (Baroody, 1988). Geary et.al. (1991) expone que el desarrollo de la combinación de estrategias y la velocidad del procesamiento para la recuperación de resultados median en beneficio de los niños con mayor rendimiento. Gathercole y Pickering (2000) sugieren que el desarrollo de estrategias flexibles para el almacenaje y la recuperación de la información, el control del 
flujo de información a través de la memoria de trabajo, la recuperación del conocimiento desde la memoria a largo plazo, o el control de la acción, la planificación, y la programación de múltiples actividades concurrentes, son funciones de la memoria de trabajo, específicamente del componente ejecutivo central.

\section{Objetivos e Hipótesis de Trabajo}

En el presente estudio proponemos estudiar el desarrollo de la memoria de trabajo en el tiempo, más específicamente la evolución de los diferentes componentes de la memoria de trabajo (bucle fonológico, ejecutivo central y agenda viso-espacial) en niños a los 6,7 y 8 años de edad, y su relación con el desempeño en tareas aritméticas como el cálculo.

Planteamos responder si, a mayor desarrollo de la memoria de trabajo hay un mejor desempeño en operaciones aritméticas, y si se encuentran diferencias en el desarrollo de dichas operaciones aritméticas.

\section{Método}

\section{Participantes}

El diseño muestral es de carácter no probabilístico, intencional. Comprendió a 103 niños de 6 años de edad (60 niños y 43 niñas) provenientes de escuelas de gestión pública (52 niños) y de gestión privada (51 niños). La muestra inicial fue dividida en dos sub muestras, según la mediana de rendimiento en memoria de trabajo. Así se conformó un grupo con mayor rendimiento en memoria de trabajo y otro de menor rendimiento en memoria de trabajo. El estudio en su tercera evaluación comprendió a 90 niños (54 niños y 36 niñas); con 47 sujetos conformando el grupo con mayor rendimiento en memoria de trabajo y 43 sujetos en el grupo con menor rendimiento en memoria de trabajo.

\section{Instrumentos}

Se seleccionaron aquellas pruebas de memoria de trabajo dentro de las válidas y confiables, aquellas que resultaron de muy fácil administración y que no requirieran de mucho tiempo, ya que la evaluación se realizó dentro del horario escolar. La administración de las pruebas estuvo sujeta a la propuesta por el manual de administración de los test. 
1) El bucle fonológico fue evaluado por el sub test complementario Retención de Dígitos de la escala de Inteligencia WISC III (Wechsler, 2003). En el test de Dígitos Directos los niños tienen que repetir series que van desde dos hasta nueve dígitos, en el mismo orden que son presentados por el examinador. El evaluador presenta las series auditivamente. Esta prueba requiere principalmente del almacenamiento de información auditiva a corto plazo en la memoria de trabajo.

2) La prueba elegida para evaluar el componente ejecutivo central fue el sub test complementario Retención de Dígitos Inversos de la Escala de Inteligencia WISC III (Wechsler, 2003).

3) En el test de Dígitos Inversos los niños deben repetir series de números no consecutivos que van desde dos hasta ocho dígitos, en orden inverso (o contrario) al presentado por el examinador. Esta prueba evalúa la habilidad para mantener una cifra en la memoria, al mismo tiempo que se la reordena. Implica la manipulación activa de la información almacenada, poniendo en funcionamiento el control ejecutivo de la memoria de trabajo.Para Baddeley (1986) los dígitos directos requieren del bucle fonológico mientras que los dígitos inversos incluyen también tareas que controla el ejecutivo central, ya que es necesario manipular activamente los dígitos para que se conserven y se hace necesaria una condición relativamente más exigente. Según Lezak, (1995) la mayoría de los autores señalan que ambas pruebas "Dígitos en orden Directo y Dígitos en orden Inverso" deben analizarse separadamente, puesto que parecen implicar diferentes componentes de la memoria a corto plazo específicamente de la memoria de trabajo.

4) Para evaluar la agenda viso-espacial se utilizó el Test de Copia y Reproducción de Memoria de Figuras Geométricas Complejas de Rey en su versión adaptada española (Rey, 1987). Esta técnica evalúa habilidades visomotoras, percepción visual y memoria visual inmediata, además de valorar habilidades de planificación y organización. El uso de la memoria de trabajo no verbal se involucra en la organización y reproducción de diseños complejos como la figura compleja de Rey-Osterrieth.

5) Para evaluar en cálculo se utilizó el Sub test Aritmética de la Escala de Inteligencia WISC III, que evalúa la resolución de problemas aritméticos (Wechsler, 2003). En este sub test el niño resuelve una serie de problemas aritméticos. Para cada ítem resuelve el problema mentalmente, sin usar lápiz y papel y responde oralmente dentro del tiempo límite. 


\section{Procedimiento}

Se realizaron evaluaciones anuales en el mismo período escolar, a mediados del año escolar. La administración de las pruebas fue individual, una vez obtenido el consentimiento de los padres o cuidadores legales de los niños para su evaluación. Siempre se otorgó libertad a los niños para participar o interrumpir la evaluación cuando ellos lo desearan.

\section{Análisis Estadístico}

Los datos obtenidos fueron procesados y analizados estadísticamente por medio del Statistical Packagefor Social Sciences (SPSS), versión 15.0. Se emplearon análisis de variancia (ANOVA y MANOVA) de medidas repetidas para evaluar los resultados.

\section{Resultados}

Evolución en el tiempo de los componentes de la memoria de trabajo

Para observar si los componentes de la memoria de trabajo mostraban cambios a lo largo del tiempo, por un lado, y si había diferencias entre los puntajes según grupo a lo largo de las tres edades estudiadas, por el otro; se realizó un análisis de variancia múltiple (MANOVA) para medidas repetidas.

En lo referente a los cambios a lo largo del tiempo según edad, se halló que los tres componentes de la memoria de trabajo cambiaban con la edad $(F$ Hotelling $(6,83)=106,71$ $p<.001$ ), lo que se debería a diferencias madurativas. A mayor edad, mayores los promedios en los componentes de la memoria de trabajo. Se detectó un efecto lineal en todos los componentes de la memoria de trabajo cuyos resultados se presentan en la tabla 1. En los tres componentes se encuentran diferencias a lo largo del tiempo.

Tabla 1. Análisis univariados intrasujetos de los componentes en el tiempo

\begin{tabular}{lll}
\hline & $\mathrm{F}(2,88)$ & $p$ \\
\cline { 2 - 3 } Bucle F. & 9,8 & $.01 *$ \\
Agenda V. & 196,78 & $.00 * *$ \\
Ejecutivo C. & 130,08 & $.00 * *$ \\
\hline$* \mathrm{p}<0.1 * * \mathrm{p}<0.5$ & &
\end{tabular}


Con relación a las diferencias según grupo de pertenencia, los resultados mostraron que los perfiles de los dos grupos eran semejantes y guardan un paralelismo. Se detectaron diferencias en las alturas de los perfiles en los tres componentes $(F$ Hotelling $(3,86)=8,36, p$ $<.001)$. Encontramos que en los tres componentes existen diferencias según grupo de pertenencia, como se puede observar en la tabla 2.

Tabla 2. Interacción grupos según rendimiento y tomas sucesivas.

\begin{tabular}{cll}
\hline & $\mathrm{F}(2,88)$ & $p=$ \\
\cline { 2 - 3 } Bucle F. & 6,55 & $.02^{* *}$ \\
Agenda V. & 3,04 & $.05 * *$ \\
Ejecutivo C. & 2,50 & .08 \\
\hline$* * \mathrm{p}<0.5$ & &
\end{tabular}

A continuación se presentan los gráficos con los desempeños de cada grupo en los diferentes componentes de la memoria de trabajo.

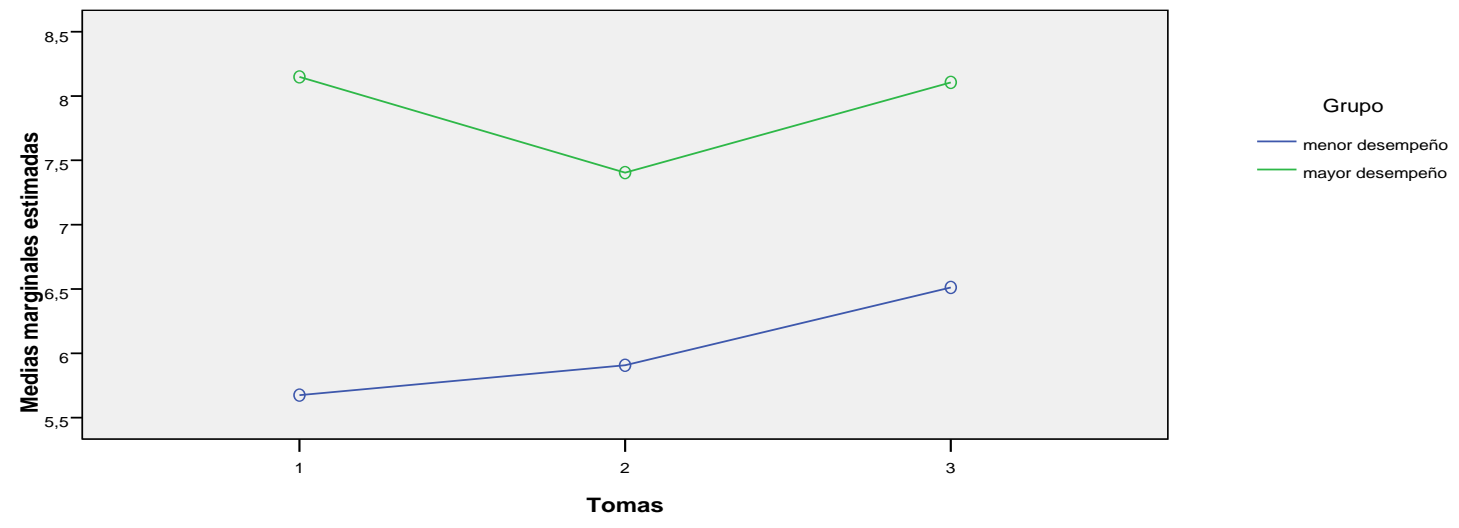

Gráfico 1. Perfiles del Bucle Fonológico a lo largo de las tres edades según grupo de pertenencia
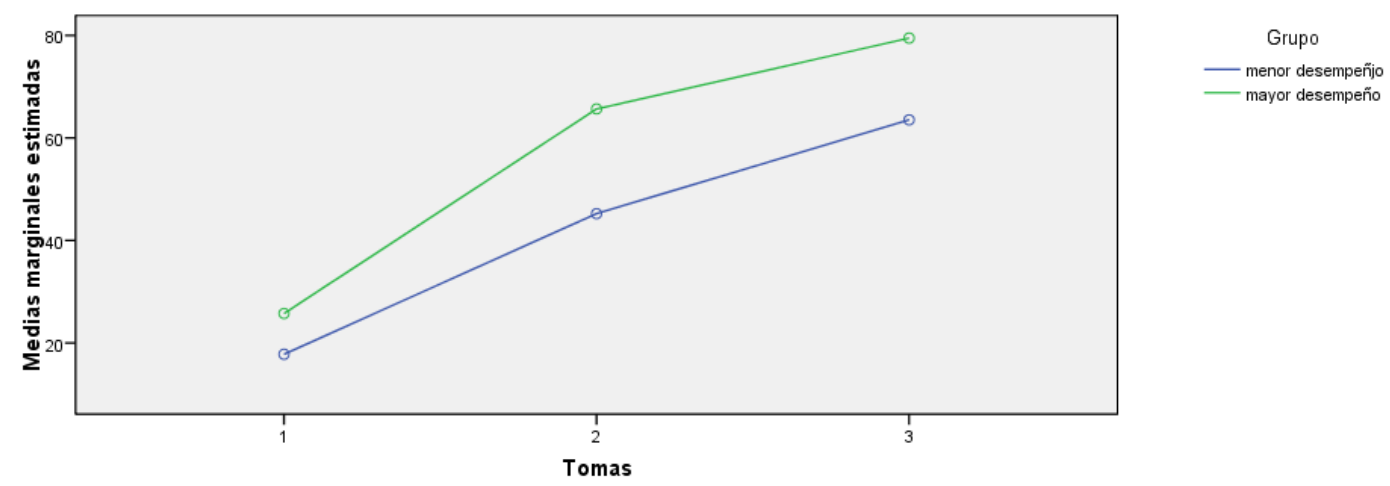

Gráfico 2. Perfiles de la Agenda Visoespacial a lo largo de las tres edades según grupo de pertenencia 


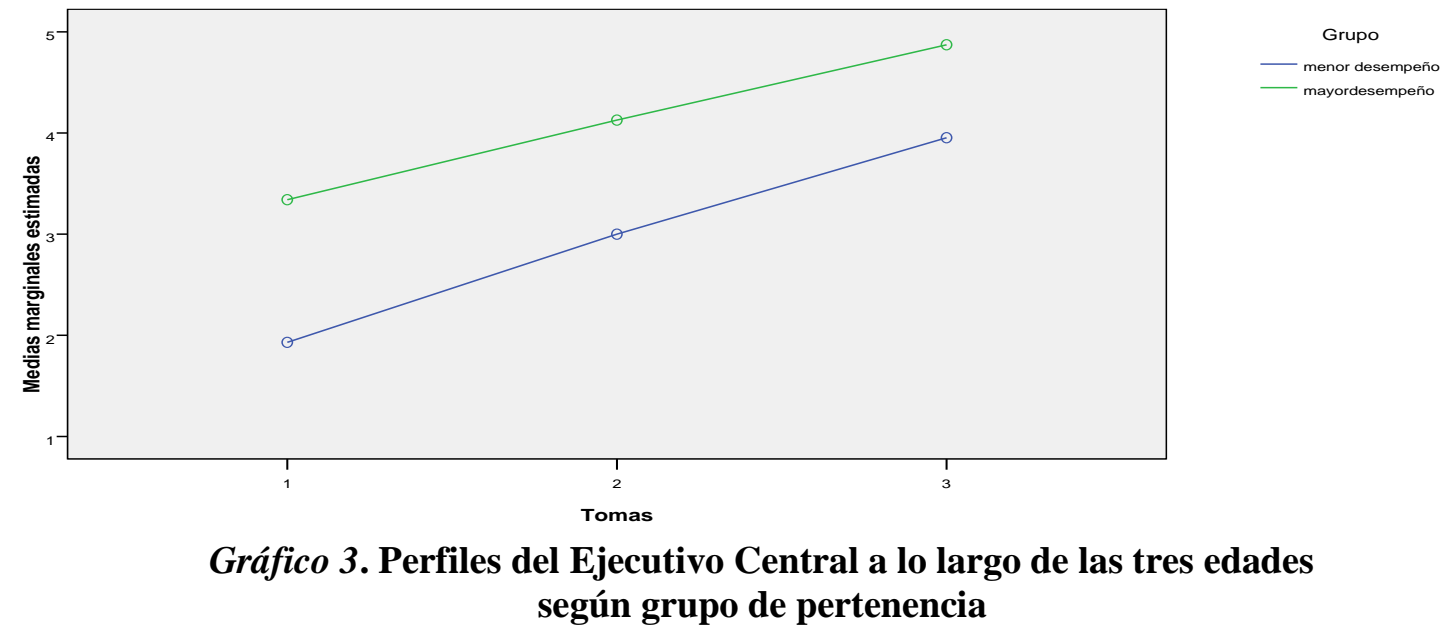

Resultados relativos a la evolución en el tiempo del cálculo y comparación según grupo

Para observar si había diferencias entre los puntajes del cálculo verbal según grupo de pertenencia a lo largo de las tres edades estudiadas, se realizó un ANOVA de medidas repetidas tomando el rendimiento en cálculo como variable dependiente. En lo referente a los cambios a lo largo del tiempo, se halló que el desempeño en el cálculo cambiaba con la edad ( $F$ $(1,88)=2257,1 p<.001)$. Esto indica que existen diferencias según la edad y que a mayor edad, mejor es el desempeño en las tareas de cálculo, independientemente del grupo de pertenencia.

Los contrastes entre las medias del desempeño en cálculo en las edades estudiadas, indicaron que el mismo cambiaba significativamente entre las edades de 6 y 8 y 7 y 8 años. En la siguiente tabla 3 se presentan los contrastes del desempeño en cálculo por edades que resultaron significativos.

Tabla 3. Pruebas de contrastes intra-sujetos en el desempeño de cálculo en el tiempo

\begin{tabular}{clrr}
\hline Medida & Edades & \multicolumn{1}{l}{$p$} \\
\hline Cálculo & & & \\
& 6 años -8 años & 2257,12 & $.00^{*}$ \\
& 7 años -8 años & 213,76 & $.00^{*}$ \\
\hline
\end{tabular}

$* \mathrm{p}<0.1$ 
En relación a las diferencias según grupo de pertenencia, los resultados mostraron que los perfiles de los dos grupos eran paralelos, pero se detectaron diferencias en las alturas de los perfiles de cálculo $(F(1,88)=10,25 p<.005)$. En ambos grupos siempre se incrementa el desempeño y siempre es menor el promedio del grupo de bajo desempeño, pero a medida que aumenta la edad se acentúan las diferencias entre los grupos, lo cual indica que hay una influencia de la interacción edad por grupo de pertenencia. El grupo de mayor desempeño muestra niveles más altos en las tres edades en comparación con el grupo de menor desempeño como se observa en el gráfico 4.

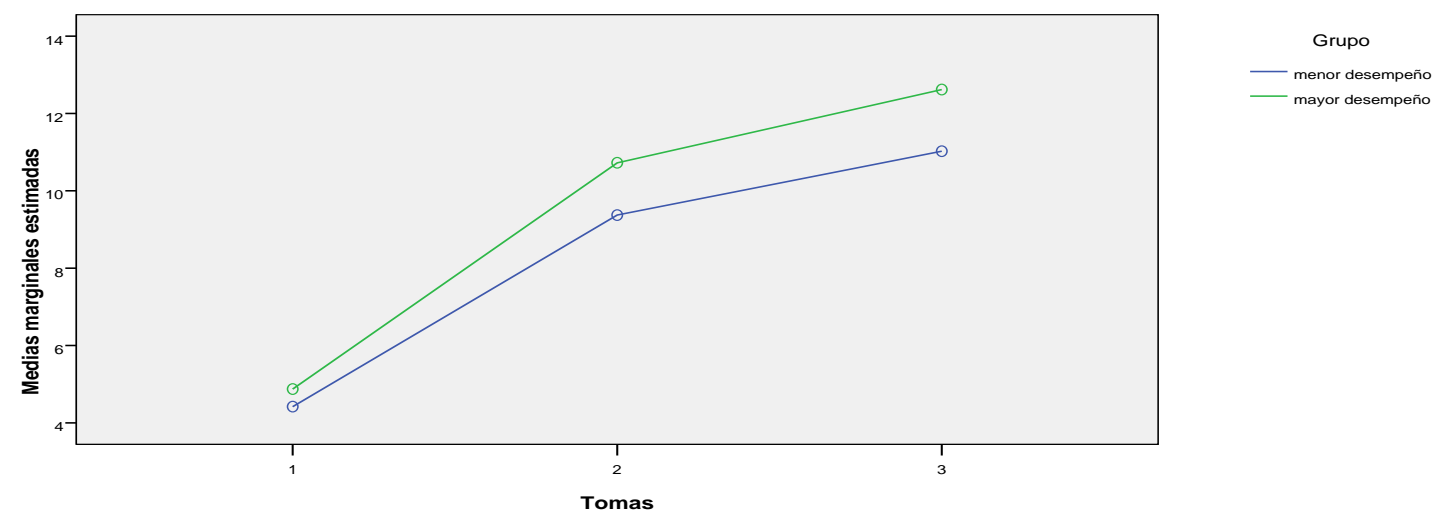

Gráfico 4. Perfiles del desempeño en cálculo a lo largo de las tres edades según grupo de pertenencia

Analizando el tipo de estrategia utilizada para resolver los cálculos de resolución de problemas, a los 8 años de edad, encontramos dos grupos: estrategias mentales y estrategias no mentales (utilización de soportes concretos). En la siguiente tabla presentamos los porcentajes de las estrategias mentales utilizadas en cada grupo.

Tabla 4. Porcentajes de Cálculo Verbal resuelto mentalmente a los 8 años según grupo de pertenencia

\begin{tabular}{lrr}
\hline $\begin{array}{l}\text { Porcentajes de estrategias mentales utilizadas en } \\
\text { la resolución del cálculo verbal }\end{array}$ & $\begin{array}{c}\text { Menor } \\
\text { desempeño } \\
\text { MT }\end{array}$ & $\begin{array}{c}\text { Mayor } \\
\text { desempeño } \\
\text { MT }\end{array}$ \\
\hline ninguna estrategia mental & 25 & 4 \\
$50 \%$ resuelto mentalmente & $56,8 \%$ & $8,7 \%$ \\
$100 \%$ resuelto mentalmente & 10 & 16 \\
& $22,7 \%$ & $34,8 \%$ \\
Total & 9 & 26 \\
& $20,5 \%$ & $56,5 \%$ \\
& 44 & 46 \\
\hline
\end{tabular}


Encontramos que la mitad de los niños con mayor desempeño emplean sólo procedimientos de tipo mental en la resolución de cálculos, mientras que en el grupo con menor desempeño sólo el $20 \%$ de los niños las utiliza como medida exclusiva de resolución.

\section{Discusión y Conclusiones}

Partiendo de que las capacidades que va adquiriendo el niño durante su desarrollo no son producto sólo de la maduración a nivel neurológico, sino que en gran medida son el resultado de la interacción con el medio, la estimulación y la educación, creemos que se puede ayudar a revertir o modificar la tendencia desfavorecida de grupos en riesgo, a través del conocimiento y estudio de los procesos involucrados como ha demostrado ser la memoria de trabajo. Nuestra intención es aportar datos al conocimiento de cómo se desarrollan los diferentes componentes de la memoria de trabajo y la incidencia sobre aprendizajes como el cálculo durante un período evolutivo vital de los aprendizajes elementales.

En primer lugar, se analizan los resultados de la evolución en el tiempo de los componentes de la memoria de trabajo y se realiza una comparación de los grupos según pertenezcan a grupos con mayor o menor rendimiento en memoria de trabajo. En segundo lugar, se analiza los resultados relativos a la evolución en el tiempo del cálculo, nuevamente se realiza una comparación según grupo de pertenencia y según las estrategias de resolución predominantemente empleada.

Con respecto al desarrollo de la capacidad de memoria de trabajo a través del tiempo encontramos que el rendimiento de todos los componentes de la memoria de trabajo presenta aumentos graduales en los tres años del estudio. Los resultados de nuestro trabajo son coherentes con los de estudios longitudinales como los de Giedd et al. (1999) y trabajos que estudiaron los incrementos en la actividad funcional relacionados con la edad a lo largo del desarrollo en niños, como Luna et. al. (2001); Klingberg, Forssberg y Westerberg (2002); Kwon, Reiss y Menon (2002); Tamm, Reiss y Menon (2002) y Rubia et.al. (2006).

Los informes ya existentes sobre las mediciones del comportamiento de la memoria de trabajo en general, plantean que la misma mejora sustancialmente durante la infancia (Gathercole, 1998, 1999), y que cada componente, como el rendimiento visoespacial (Logie y Pearson 1997; Pickering y Gathercole et. al., 2001) y el funcionamiento del bucle fonológico se incrementan con la edad (Gathercole et al., 2004). 
La neuropsicología nos brinda información sobre cambios estructurales y desarrollos progresivos de las áreas cerebrales involucradas con la memoria de trabajo, que experimentan una maduración formidable en sus funciones durante la infancia. Se puede decir que este desarrollo se caracteriza por un lado, por una reducción de la densidad sináptica y neuronal, eliminando selectivamente las sinapsis menos relevantes y produciéndose un crecimiento de las dendritas. Pero también es un período en el que la mielinización aumenta la velocidad de conducción nerviosa (Baumann y Pham-Dinh, 2001) y se produce un aumento del volumen de materia blanca en la corteza prefrontal que proporciona una base estructural para las funciones cognitivas (Bartzokis, Beckson, Lu, Nuechterlein, Edwards y Mintz, 2001). Así, la mielinización contribuye en gran medida a mejorar la funcionalidad del cerebro, ya que produce un incremento en la velocidad de conducción de los impulsos nerviosos (Klingberg, Vaidya, Gabrieli, Moseley y Hedehus, 1999). Con respecto al desarrollo de operaciones de cálculo durante los tres años, como era de esperarse, encontramos que existen cambios en los desempeños en cálculo. Al tratarse de una población escolarizada, año a año y progresivamente, fueron adquiriendo contenidos aritméticos más complejos promoviendo la conformación de redes neuronales a través de la experiencia y de la maduración progresiva de las áreas cerebrales involucradas que posibilitan la realización de actividades cognitivas cada vez más complejas.

Al comparar el conocimiento aritmético alcanzado según el grupo de pertenencia, resulta llamativo observar cómo comienzan la escolaridad con una discrepancia en los logros que se incrementa a medida que pasan los años, encontrándose mayores rendimientos aritméticos en los niños con mayor rendimiento en memoria de trabajo.

Según Geary (1991), el desarrollo de la combinación de estrategias y la velocidad del procesamiento para la recuperación de resultados median en beneficio de los niños con mayores rendimientos en cálculo. Teniendo en cuenta esta afirmación y a partir de los resultados obtenidos, nos pareció interesante explorar las estrategias que utilizaban en cada grupo a los 8 años, edad donde se encuentran las mayores diferencias en los desempeños. Es de esperar que las estrategias varíen en función de la edad, en busca de la rentabilidad. Por lo tanto, al comienzo de la escolaridad las estrategias preferidas por los niños son las que se acercan más a lo manipulativo, pero a medida que el niño adquiere más seguridad opera mentalmente tendiendo a elegir estrategias que resultan más eficaces en cuestión de velocidad de resolución. Encontramos que la mitad de los niños del grupo con mayor desempeño en memoria de trabajo utilizaban sólo estrategias mentales, mientras que la mitad de los niños del grupo con me- 
nor desempeño en memoria de trabajo no usa ninguna estrategia mental para la resolución del cálculo.

Neuroanatómicamente vimos que las operaciones aritméticas tienen un inicio frontal, que progresivamente se va especializando en un procesamiento más parietal, al automatizarse las relaciones entre los símbolos y las magnitudes que ellos representan. Posiblemente los niños del grupo con menor desempeño en memoria de trabajo, al requerir mayor control atencional, demandan a los sistemas frontales mayor actividad. A medida que se realizan cálculos se aumenta la fuerza asociativa entre nodos de hechos aritméticos y nodos de respuestas y se va produciendo la automatización de las operaciones aritméticas elementales, lo cual supone un importante ahorro cognitivo (Baroody, 1988). El ahorro cognitivo posibilita que la memoria de trabajo pueda atender a otras actividades más exigentes o desafiantes desde el punto de vista cognitivo, como el cálculo mental. Podría decirse que la mayoría de los niños del grupo con bajo desempeño en memoria de trabajo requieren de las funciones de ésta para los cálculos elementales, debido a que poseen escasa fuerza asociativa internodal por lo que frecuentemente deben recurrir a estrategias más manipulativas o de soporte concreto, en detrimento del tiempo de ejecución, lo que hace más difícil que lleguen a alcanzar niveles de cálculos más complejos.

De todo lo expuesto en el trabajo podemos concluir que la memoria de trabajo se desarrolla evolutivamente, como también los conocimientos aritméticos según pasan los años. La discrepancia en los rendimientos en aritmética, según se tenga alto y bajo desempeño en memoria de trabajo, se acrecienta con el pasar del tiempo. La memoria de trabajo tiene incidencia en las estrategias utilizadas para la resolución de problemas aritméticos, encontrándose estrategias de resolución predominantemente mentales en el grupo con alto rendimiento en memoria de trabajo. En el grupo con menor rendimiento en memoria de trabajo, se utilizan estrategias sujetas a mayor comprobación y sustento concreto en detrimento de la velocidad y precisión de las resoluciones aritméticas. Las estrategias de resolución tendientes a la rentabilidad y ahorro cognitivo se encuentran en los niños con mayores rendimientos. La utilización de estrategias sujetas a la memoria de trabajo difiere según la modalidad de resolución empleada, sea mental o manipulativa.

Posiblemente los hallazgos presentados en la presente investigación podrían estar respaldados y complementados mediante técnicas de neuroimágen, que dé cuenta de estos cambios y relaciones neuropsicológicas, convirtiéndose en una limitación del estudio. Sería inte- 
resante seguir profundizando en el estudio de la relación entre la atención y la memoria de trabajo, dada su importancia para el aprendizaje. Por otra parte, podría seguir estudiándose la relación entre las funciones cognitivas, las competencias lingüísticas y las matemáticas, por su alto impacto en el desarrollo neurocognitivo del niño. Asimismo, podrían utilizarse otras técnicas de evaluación para medir y comparar las funciones estudiadas. 


\section{Referencias}

Ansari, D., Gracia, N., Lucas, E., Halmon, K. y Dhital, B. (2005). Neural correlates of symbolic number processing in children and adults. Brain Imaging, 16, 1769-73. doi:10.1097/01.wnr.0000183905.23396.f1

Baddeley, A D. (1986). Working Memory. Oxford: Oxford Univ. Press.

Baddeley, A. D. (1992). Working memory. Science, 255, 556-559. doi:10.1016/10538100(92)90037-B

Baddeley, A. y Hitch, G. (1974). Working memory. In G.A. Bower. The Psychology of Learning and Motivation (pp. 47- 89). New York: Academic Press. doi:10.1016/S0079-7421(08)60452-1

Baroody, A. (1998). El pensamiento matemático de los niños. Aprendizaje Visor, Madrid.

Bartzokis, G., Beckson, M., Lu, P., Nuechterlein, K., Edwards, N. y Mintz, J. (2001). Agerelated changes in frontal and temporal lobe volumes in men: a magnetic resonance imaging study. Arch Gen Psychiatry, 58, 461-5. doi:10.1001/archpsyc.58.5.461

Baumann, N. y Pham-Dinh, D. (2001). Biology of oligodendrocyte and myelin in the mammalian central nervous system. Physiol Rev, 81, 871-927.

Gathercole, S.E. (1998). The development of memory. J Child Psychol Psychiatry, 39, 3-27. doi:10.1017/S0021963097001753

Gathercole, S.E. (1999). Cognitive approaches to the development of short-term memory. Trend Cogn Sci, 3, 410-9. doi:10.1016/S1364-6613(99)01388-1

Gathercole, S. E. y Pickering, S. J. (2000). Working memory deficits in children with low achievements in the national curriculum at 7 years of age. British Journal of Educational Psychology, 70, 177-194. doi:10.1348/000709900158047

Gathercole, S.E., Pickering, S.J., Ambridge, B. y Wearing, H. (2004). The structure of working memory from 4 to 15 years of age. Developmental Psychology, 40, 177-90. doi:10.1037/0012-1649.40.2.177

Geary, D.C. y Brown, S.C. (1991). Cognitive addition: Strategy choice and speed of processing differences in gifted, normal, and mathematically disabled children. Developmental Psychology, 27 (3), 398-406. doi:10.1037//0012-1649.27.3.398

Klingberg, T., Vaidya, C.J., Gabrieli, JD., Moseley, M.E. y Hedehus, M. (1999). Myelination and organization of the frontal white matter in children: a diffusion tensor MRI study. Neuroreport, 10(13), 2817-21. doi:10.1097/00001756-199909090-00022 
Klingberg, T., Forssberg, H. y Westerberg, H. (2002). Increased brain activity in frontal and parietal cortex underlies the development of visuospatial working memory capacity during childhood. J Cogn Neurosci, 14, 1-10. doi:10.1162/089892902317205276

Kwon, H., Reiss, A.L. y Menon, V. (2002). Neural basis of protracted developmental changes in visuo-spatial working memory. Proc Natl Acad Sci U S A, 99, 13336-41. doi:10.1073/pnas.162486399

Lee, K.M. y Kang, S.Y. (2002). Arithmetic Operation and Working Memory: Differential Suppression in Dual Task. Cognition, 83, B63-B68. doi.org/10.1016/S00100277(02)00010-0

Lezak, M.D. (1995). Neuropsychological Assessment (3rd. Ed.). Oxford: Oxford University Press (original publicate in 1976).

Logie, R.H. y Pearson, D.G. (1997). The inner eye and the inner scribe of visuo-spatial working memory: evidence from developmental fractionation. Eur J Cogn Psychol, 9, 24157.

Luna, B., Thulborn, KR, Munoz, D.P., Merriam, EP., Garver, KE y Minshew, NJ. (2001). Maturation of widely distributed brain function subserves cognitive development. Neuroimage, 13, 786-93. doi.org/10.1006/nimg.2000.0743

Müller, N.G y Knight, R.T. (2006). The functional neuroanatomy of working memory: contributions of human brain lesion studies. Neuroscience, 139, 51-8. doi:10.1016/j.neuroscience.2005.09.018

Pickering, S.J., Gathercole, S.E., Hall, M. y Lloyd, S.A. (2001). Development of memory for pattern and path: further evidence for the fractionation of visuo-spatial memory. Q $\mathrm{J}$ Exp Psychol A, 54, 397-420. doi.org/10.1080/713755973

Rey, A. (1987). Test de copie et de reproduction de mémoire de figures géometriques complexes. Test de copia de una figura compleja. Madrid: TEA.

Rubia, K., Smith, AB., Woolley, J., Nosarti, C., Heyman, I. y Taylor, E. (2006). Progressive increase of frontostriatal brain activation from childhood to adulthood during eventrelated tasks of cognitive control. Hum Brain Mapp, 27, 973-93. doi.org/10.1002/hbm.20237

Serra-Grabulosa, J. M., Adan, A., Pérez-Pàmies, M., Lachica, J. y Membrives, S. (2010). Bases neurales del procesamiento numérico y del cálculo. Rev Neurol, 50 (1), 39-46.

Siegler, R. S. (1987). The perils of averaging data over strategies: An example from children's addition. Journal of Experimental Psychology, 116, 250-264. doi.org/10.1037/00963445.116.3.250 
Siegler, R. S. y Shrager, J. (1984). Strategy choice in addition and subtraction: How do children know what to do? En C. Sophian Ed. Origins of cognitive skills (pp. 229-293). Hillsdale, NJ: Erlbaum.

Tamm, L., Menon, V. y Reiss, A.L. (2002). Maturation of brain function associated with response inhibition. $J$ Am Acad Child Adolesc Psychiatry, 41, 1231-8. doi.org/10.1097/00004583-200210000-00013

Unsworth, N. y Engle, R. (2007). The Nature of Individual Differences in Working Memory Capacity:Active Maintenance in Primary Memory and Controlled Search From Secondary. Memory Psychological Review, 114(1), 104-132. doi.org/10.1037/0033295X.114.1.104

Wechsler, D. (2003). Wechsler Intelligence Scale for Children: Fourth edition. San Antonio, TX:The Psychological Corporation. 